\title{
PROOF OF A THEOREM OF JACOBI
}

N. J. FINE

$\mathrm{Jacobi}^{1}$ proved the following theorem:

If $G(z)$ is defined in $[-1,1]$, then

$$
\begin{aligned}
I_{n} & \equiv \int_{0}^{\pi} G(\cos x) \cos n x d x \\
& =\frac{1}{1 \cdot 3 \cdot 5 \cdots(2 n-1)} \int_{0}^{\pi} G^{(n)}(\cos x) \sin ^{2 n} x d x .
\end{aligned}
$$

His first proof, for the case in which $G(z)$ may be expanded in a power series, depends on the formula

$$
\begin{aligned}
\int_{0}^{\pi} \cos ^{p} x & \cos n x d x \\
& =\frac{p(p-1) \cdots(p-n+1)}{1 \cdot 3 \cdot 5 \cdots(2 n-1)} \int_{0}^{\pi} \cos ^{p-n} x \sin ^{2 n} x d x,
\end{aligned}
$$

which is itself a special case of (1). His second proof, which assumes nothing about the derivatives of $G(z)$ of order exceeding $n$, depends on the lemma

$$
\frac{d^{n-1}}{d z^{n-1}}\left(1-z^{2}\right)^{(2 n-1) / 2}=(-1)^{n-1} 1 \cdot 3 \cdot 5 \cdots(2 n-1) \frac{\sin n x}{n},
$$

where $z=\cos x$. He points out that (3) may also be deduced from (1).

We offer here a short proof by induction which does not involve previous knowledge of (2) or (3). For $n=1$ the theorem is seen to be true by an integration by parts. Now

$$
\begin{aligned}
I_{n+1} & =\int_{0}^{\pi} G(\cos x) \cos x \cos n x d x-\int_{0}^{\pi} G(\cos x) \sin x \sin n x d x \\
& =\int_{0}^{\pi} G(\cos x) \cos x \cos n x d x-n \int_{0}^{\pi} G_{1}(\cos x) \cos n x d x
\end{aligned}
$$

by integration by parts, where $G_{1}(z)$ is an integral of $G(z)$. Applying the induction hypothesis to $F(z)=z G(z)-n G_{1}(z)$ and observing that $F^{(n)}(z)=z G^{(n)}(z)$, we get

Presented to the Society, February 25, 1950; received by the editors August 16, 1949.

1 C. G. J. Jacobi, J. Reine Angew. Math. vol. 15 (1836) pp. 1-26. 


$$
\begin{aligned}
I_{n+1} & =\int_{0}^{\pi} F(\cos x) \cos n x d x \\
& =\frac{1}{1 \cdot 3 \cdot 5 \cdots(2 n-1)} \int_{0}^{\pi} G^{(n)}(\cos x) \sin ^{2 n} x \cos x d x .
\end{aligned}
$$

Another integration by parts yields

$$
I_{n+1}=\frac{1}{1 \cdot 3 \cdot 5 \cdots(2 n+1)} \int_{0}^{\pi} G^{(n+1)}(\cos x) \sin ^{2 n+2} x d x,
$$

and the theorem is proved.

University of Pennsylvania 